\title{
Optogenetics and Controlling the Human Mind
}

\author{
Michael Guynn ${ }^{1}$ a \\ ${ }^{1}$ Psychology, Dixie State University \\ Keywords: optogenetics, mind control, operant conditioning, brain stimulation, CRISPR \\ https://doi.org/10.36898/001c.28096
}

Curiosity: Interdisciplinary Journal of Research and Innovation

From the dawn of history to modern times humans have been using knowledge of neural structures to alter behavior (Faria, 2013). In modern times brain stimulation experiments have been conducted on animals and even humans to control the mind (Marzullo, 2017; Bishop et al., 1963). Behavioral psychologist B.F. Skinner proposed that all behavior can be controlled using rewards and punishments (Schultz \& Schultz, 2019). A new technology in neural engineering known as optogenetics uses CRISPR Cas- 9 to genetically modify human neurons to express photosensitive opsins and thus fire when stimulated by certain light wavelengths (Boyden, 2011). Optogenetics offers greater spatial and temporal control of brain activity than current technologies like transcranial magnetic stimulation or psychopharmacological drugs (Williams and Entcheva, 2015; Deisseroth et al., 2006; Shao et al., 2018). The ethics of potential side effects, invasiveness, and abuse should be taken into consideration before human trials begin in the near future (Mathews, 2011; Gilbert, Harris, \& Kidd, 2021).

\section{Introduction}

With 86 billion neurons and more synapses than stars in the milky way galaxy, the human brain is a fascinating structure (Herculano-Houzel, 2009). Many psychologists would likely agree with neuroscientist Michio Kaku that the human brain is the most complicated object in the known universe (Bartucca, 2018). Although interest in discovering the mysteries of the brain is as old as human history, relatively little is known about its complicated inner workings. However, technological development in the 21 st century is allowing neuroscientists to understand increasing details about the brain's structure and function.

The human connectome project is a highly ambitious neuroscientific study that is currently using advanced brain-imaging technologies to develop the most detailed maps of neuronal structures to date (Van Essen et al., 2012). Using MRI the human connectome project has already identified 180 distinct areas or modules in the cerebral cortex alone. One day science may provide a complete knowledge of the brain's myriad structures and their connections.

Parallel to humanity's search for knowledge is the desire to control the brain. From ancient brain surgeries to lobotomies in the 20th century, humans have used knowledge of neuronal structures to manipulate behavior (Faria, 2013). As the human connectome project and other studies reveal details about the brain's structure, so are technological advances allowing humans increasing 
power to harness neural activity. There is much excitement over Elon Musk's neuralink technology which is projected to begin human trials by 2023. Musk's neuralink device will be the first mainstream BMI (brain-machine interface). Recording from 1024 microelectrodes implanted in the motor cortex by a surgical robot, the neuralink device will allow people to control smartphones and other devices by thought alone. On the neuralink website is this statement: As our technology develops, we will be able to increase the channels of communication with the brain, accessing more brain areas and new kinds of neural information (www.neuralink.com). Neuralink and other technologies will expand their influence throughout the brain, but BMIs may only be the beginning.

A new technology in the field of neuroscience with massive potential is optogenetics. Optogenetics uses CRISPR Cas-9 to genetically modify human neurons to fire when stimulated by light alone. Optogenetics will provide a level of understanding and control over the human brain yet unparalleled in neuroengineering. In this paper we will discuss historical instances of mind control, the mechanisms used to manipulate behavior, the emergence of optogenetic technologies, and the ways in which optogenetics may be used to control behavior.

\section{History of Mind Control}

From the very dawn of history to modern times humans have been using surgical methods to alter behavior. Dating back to the neolithic age, ancient South Americans and Europeans alike have used a primitive surgery known as trephination. Trephination involved slicing off sections of the calvarium in order to release spirits trapped within the brain. Trephination may have been used for treatment of intractable epilepsy and demonic possession (Faria, 2013). While these primitive surgeries may be the precursors of modern neuromedicine, widespread manipulation of the brain to change behavior did not emerge until the 20th century.

A specific incident known as the American Crowbar Case spurred the rise of modern psychosurgery. Phineas Gage, a railroad worker, was severely wounded when an accidental explosion shot a tamping iron through his head, almost completely destroying his left frontal lobe. Although Gage survived the ordeal, his personality did not. Gage's behavior changed from personable and responsible to impulsive and insensitive. Fascinated by Gage's dramatic shift in behavior, scientists began studying the frontal lobes (Faria, 2013).

Studies done on chimpanzees revealed that lesioning of the prefrontal cortex could decrease aggressive behavior and increase docility in these animals. The overcrowded mental Asylums of the mid-1900s began to use similar forms of psychosurgery to sedate disturbed patients. The most popular procedure, the transorbital leucotomy, was invented by Dr. James Watts and involved inserting an ice pick through the space above the eyeball into the brain where it was rotated to sever white-matter connections beneath the frontal lobe. 60,000 
frontal lobe surgeries were completed in a timespan of twenty years (1936-1956), many of them on mental patients who did not or could not give consent (Faria, 2013).

Early psychosurgical treatments such as lobotomies seem unethical and even barbaric by current standards of mental health. However, other instances of neural manipulation continued into the 70s. The CIA project MKULTRA, composed of 149 subprojects, was a massive covert study that used various methods of behavioral manipulation on the American People. Beginning in 1953 and revealed to the public in 1975, project MKULTRA used LSD, chemically-induced comas lasting as long as 60 days, dangerous levels of electroshock therapy, pharmacological paralysis, and psychic driving (a combination of drugs and tape-recordings) to control the human mind. Many of these experiments were conducted without the participants' knowledge or consent (Neely, 2021).

Although the full range of mind-control methods used across history is beyond the scope of this paper, the development of brain-stimulation technology is of great interest. One of the first instances of mind control using brain-stimulation was an experiment done by Dr. Jose Delgodo in the 1960s using Spanish bulls and radio waves. After anesthetizing a number of bulls, Delgado surgically implanted up to 28 electrodes in brain areas such as the primary motor cortex, thalamus, and basal ganglia. Using a handheld controller, Delgado was able to send radio waves to the bull's electrodes which generated electrical impulses in their brains. Using his device, Delgado could stop a bull in full charge just 2-3 meters from hitting him using radio-induced stimulation. When the stimulation ended the bull resumed full charge, ramming against the wooden barrier where Delgado had been standing only moments before (Marzullo, 2017).

Although early experiments like Delgado's were done on animals, one study in 1963 titled Intracranial Stimulation in Man was performed on a human. The patient was a 35-year old man who had been institutionalized for nine years with severe schizophrenia. After craniotomy, electrodes were surgically implanted in the man's amygdala, thalamus, and hypothalamus. Wires ran from the electrodes to a pair of switches in front of the patient. By pressing the switches the patient could therefore stimulate his own brain. One switch delivered a mild electric pulse which produced rewarding or pleasurable psychological effects. The other switch delivered a slightly stronger pulse to the same brain areas which produced aversive psychological effects. The patient, upon pressing both the levers, quickly adapted to pressing only the lever that produced pleasant stimulation of his brain. The patient began to show signs of addiction to this stimulation, even denying his basic need of hunger to press the lever. The authors of the study noted: "It is of interest that the introduction of an attractive tray of food produced no break in responding although the subject had been without food for seven hours." Although the experiment was highly invasive there is no mention in the paper of informed consent (Bishop et al., 
1963). This study, while being obviously unethical by current standards, opens up a discussion about how brain stimulation might control behavior through operant conditioning.

\section{Brain Stimulation and Operant Conditioning}

The readers of Anthony Burgess's classic novel $A$ Clockwork Orange may remember the iconic sequence in which the main character Alex is strapped to a chair and forced to watch extremely violent films. Before the films begin, Alex is administered a drug that is designed to make him extremely ill. As the drug's effects begin to take hold, brain monitoring technologies relay Alex's mental state to a team of researchers which modulate the violent images so that Alex begins to pair concepts of violence with nausea. This Ludovico treatment is so effective that Alex can no longer imagine violent acts without feeling ill after he is released (Servitje, 2018).

Although the Ludovico treatment itself is science fiction, there are a number of modern treatments known as aversion therapy that operate with similar mechanisms. A number of emetic drugs such as apomorphine and disulfiram work by stimulating nausea-inducing areas of the brain. The emetic center of the brain, the nucleus tractus solaris (NTS) located in the brainstem medulla, is the most obvious target for these drugs. Aversion therapy works by pairing feelings of nausea with any unwanted behavior. For example, the drug disulfiram interferes with the metabolism of ethyl alcohol and produces severe sickness and discomfort each time an alcoholic beverage is consumed. Thus, patients of disulfiram develop a strong aversion to anything connected to alcohol (MacDougall \& Sharma, 2020; Servitje, 2018).

Although discomfort may be a powerful motivator, behavior can also be modified with psychological pleasure. The nucleus accumbens (NAcc), known as the brain's reward center, is the center of addiction and habit formation in the brain. As part of the mesolimbic reward pathway between the prefrontal cortex and the emotional limbic system, the NAcc facilitates subjective experiences of pleasure when introduced to favorable stimuli. Studies have shown that stimulation of the NAcc can effectively increase feeding behavior of rats (Baumgartner et al., 2020). Additionally, deep brain stimulation technologies that increase neural firing in the NAcc are approved for the treatment of depression in humans by relieving symptoms of anhedonia (lack of pleasure) (Delaloye \& Holtzheimer, 2014).

These behavior-modifying treatments (NTS stimulation for aversion and NAcc stimulation for reward) can be seen as modern correlates of the behavioral psychologist B.F. Skinner's theory of operant conditioning. Skinner theorized that all human behavior can be controlled via a system of rewards and punishments. A reward is anything that increases behavior whereas a punishment is anything that decreases behavior. Skinner demonstrated his theories by training animals to do complex tasks using nothing but rewards such as food pellets and punishments such as electric shocks. Skinner's behavioral therapies extended to the human world for the treatment of depression, reward systems for school children, and teaching social behaviors 
for individuals with autism (Shultz \& Schultz, 2019). By stimulating areas like the NTS and NAcc, one could theoretically control any human behavior via operant conditioning. One radical new technology is emerging in the field of neuroengineering that may be able to modify human behavior in new, unparalleled ways.

\section{Optogenetics}

\section{Introduction: Controlling the Mind with Light}

CRISPR Cas-9 is a groundbreaking gene-modifying technology that uses natural virus-defence mechanisms from bacteria. CRISPR uses two components to edit genes: a snippet of guide RNA and a Cas-9 nuclease. The guide RNA is first inscribed with a specific genetic sequence of nucleotides which the Cas- 9 nuclease then uses to find a corresponding section of DNA. Once Cas-9 finds the target DNA it cuts the sequence, thus silencing its genetic expression or inserting a new genetic sequence for modified expression (Shao et al., 2018). A new technology in the field of neuroengineering, coined optogenetics, uses CRISPR Cas-9 to express opsins in human neurons.

Opsins are light-sensitive proteins that use photopigments to capture photons from sunlight. Once a specific wavelength of light is captured by an opsin's photopigment the opsin changes shape to open an ion channel to which it is attached. This ion channel then allows negative or positively charged ions to pass into or out of the opsin's host cell, altering the intracellular chemical gradient and changing the cell's behavior (Boyden, 2011).

Optogenetics uses CRISPR Cas- 9 to insert the genetic sequence for opsins into the DNA of selected human neurons. CRISPR complexes holding the target genetic sequences are injected via micro-syringe into the selected neuron for genetic modification. Once CRISPR adds the opsin sequence to the neuron's DNA, opsins are genetically expressed on the cell membrane of the neuron. When these opsins are illuminated with their target wavelength they open their ion channels and change neuronal activity (Boyden, 2011).

Two kinds of opsins have been discovered so far for the use of optogenetics: neuronal exciters and neuronal silencers. The channelrhodopsin- 2 opsin, extracted from the DNA of a species of green algae, is activated by blue light. Once activated, channelrhodopsin-2 allows positively-charged ions such as sodium to flow into the neuron which depolarizes the cell and causes it to fire. Thus, channelrhodopsin-2 can be used to stimulate neural activity. Conversely, another opsin called archaerhodopsin is extracted from a species of archaea and responds to light in the green/yellow spectrum. Once activated, archaerhodopsin pumps protons out of the neuron and thus hyperpolarizes it, removing the chance that the neuron will depolarize and fire. Thus, archaerhodopsin can be used to silence neural activity (Boyden, 2011).

Optogenetics has several advantages over other forms of neural-stimulation including pharmacology and electrical stimulation. Pharmacological drugs act systemically due to blood-stream distribution and thus have many side effects. Although certain drugs may change electrochemical activity in target areas of the brain, they also act on many untargeted areas of the brain. Additionally, 
drug metabolism and distribution may take minutes, hours, or even weeks to produce noticeable effects. Thus, pharmacological stimulation of the brain suffers from extremely low temporospatial resolution (it poorly mimics natural neuronal behavior). New treatments utilizing electrical stimulation of the brain tend to have much better temporospatial resolution than pharmacological drugs. The microelectrodes of deep brain stimulation, for example, offer accurate stimulation of target brain areas with much less, if any, side effects. However, even deep brain stimulation activates millions of neurons at a time and cannot activate highly localized neural pathways or groups of neurons. Additionally, because electrodes deliver electrical impulses without feedback from the target neurons, electrical brain stimulation does not accurately mimic neuronal activity which is highly specific to each neuron's phenotype. Optogenetics, on the other hand, has immaculate temporospatial resolution. Highly-specific groups of neurons can be targeted, even at the level of a single neuron, because only genetically modified neurons will express opsins and respond to light stimulation (high spatial resolution). Additionally, optogenetic neurons will not only activate the instant they are exposed to the target light source (high temporal resolution) but each optogenetic neuron will function naturally in coherence with its phenotype. Thus, optogenetics accurately mimics natural brain activity on both temporal and spatial levels (Deisseroth et al., 2006; Shao et al., 2018; Williams \& Entcheva, 2015).

The applications of optogenetics are staggering. Using optogenetics to silence or excite highly specific groups of neurons will allow us to understand the structure and function of the human brain on a microlevel. Brain-mapping projects such as the human connectome project may use optogenetics to discover thousands of new areas below the level of MRI-detection. Unlike psychosurgery, optogenetics is reversed as soon as the neuro-opsins are deprived of their target lightsource, meaning human trials would be relatively harmless and without permanent effects. Technologies such as deep brain stimulation may also be replaced by optogenetics which could accurately stimulate or silence areas of the brain involved in brain disorders. Optogenetics may be used as the primary treatment for depression, anxiety, and a host of other neurological diseases (Deisseroth et al., 2006). Although there is much excitement over the potential for optogenetic technologies, there are a number of ethical considerations for its future use in human beings.

\section{Optogenetics and Ethics}

Earlier we discussed how selective stimulation of the NTS and the NAcc could be used as punishment and reward mechanisms to condition a human being for any set of behaviors. Because optogenetics allows instant and selective stimulation of any brain area, optogenetic neurons in the NTS and NAcc could be stimulated to decrease or increase desired behaviors. A study by Baumgartner et al. (2020) tested optogenetic stimulation in mice. The study used CRISPR to implant opsins in different areas of the NAcc and found that, depending on the location of stimulation, both desire and dread could be reliably induced by optogenetic stimulation (Baumgartner et al., 2020). It 
appears that operant conditioning is already possible with optogenetics, but how could this technology be used similarly in humans? While we are far from totalitarian governments implanting optogenetics for societal mind control (Gilbert et al., 2021), there are a number of ethical concerns for the future use of CRISPR and optogenetic technologies.

Because of CRISPR's potential to treat over 10,000 genetic diseases in humans and its relative ease of use it may become an invaluable asset to biomedicine in the near future. However, concerns are raised due to implications for overuse of the technology or its use before sufficient clinical studies have been completed as in the case of professor Jian-kui He (Memi et al., 2018). There has already been intense debate about the ethics of CRISPR Cas-9 following Chinese professor Jian-kui He's experiment using gene-editing on twin infants to remove AIDs susceptibility (Ye et al., 2019). This experiment clearly violated a number of ethical guidelines including informed consent. The possibilities of so-called "designer babies" as a result of unregulated CRISPR use are troubling.

Optogenetics, although yet to see human trails, has garnered ethical discussion as well. Research involving another brain-stimulating technology, deep brain stimulation (DBS), has already unearthed a host of issues associated with invasive brain implants. Studies have found that because of DBS's sudden onset of stimulation, many patients feel that its effects are overly mechanical and can even feel disruptive to a person's sense of cognitive authenticity (de Haan et al., 2017; Mathews, 2011). Another study found that DBS stimulation decreased patient's levels of self-reflection and motivation (Pham et al., 2015). Although optogenetics offers more precise and sophisticated stimulation than DBS (Kravitz et al., 2010), there is still potential for disruption of subjective feelings of self such as narrative continuity and identity (Mathews, 2011). Indeed, although the effects of optogenetics are reversible, the implantation of viral DNA into human neurons is not. Both subjective personality change following optogenetic stimulation and the invasive nature of the technology are key ethical dilemmas that must be discussed before human trials are considered (Gilbert et al., 2014).

\section{Theoretical Abuse of Optogenetics}

Although the chances of actual mind-control using optogenetics are currently improbable, future abuse of this technology is theoretically possible. Beyond remote stimulation of reward and punishment pathways is the potential for stimulation of diverse brain areas related to perception and behavior. Studies have shown that electrical stimulation of the medial superior temporal lobe changes perception of direction in macaque monkeys (Britten, 2002). Other areas, such as the fusiform gyrus, parahippocampal place area, occipital lobe, Wernicke's area, and the insula are responsible for various other types of perception. With hundreds of different opsins, each responsive to one of a hundred specific light sources, a human being's core perception of 
reality could be guided, manipulated, or distorted at will. Optogenetic-humans may even be lobotomised by selective silencing of the prefrontal cortex using archaerhodopsin.

Additional technologies in combination with optogenetics may cement control over the optogenetic-human being. Using an electroencephalogram, researchers were able to visualize and encode dozens of neural firing patterns using groups of voxels (pixels representing brain activity in a digital program). These voxel patterns were recorded over the primary visual cortex in patients who were drifting off to sleep (bypnagogia). By feeding the coded patterns into a machine-learning program, the researchers were able to predict what the participants were dreaming with $60 \%$ accuracy. As EEGs and voxel-analysis become more sensitive, the accuracy of mind-reading technologies will increase. Thus, future multi-layered systems involving feedback between voxelanalysis and optogenetic stimulation could predict and control behavior with the speed and accuracy of natural thought.

While malignant uses of optogenetics and other technologies are extremely improbable in the present, the potential for future abuse remains a topic for consideration and caution as optogenetics approaches clinical trials in human beings. Studies such as the CIA's project MKULTRA have shown how powerful and damaging technology can become in the hands of powerful, unethical organizations (Neely, 2021).

\section{Conclusion}

In this paper we discussed the history of mind-control techniques such as the frontal lobotomy, described forms of operant conditioning that may be used to control behavior, outlined the emerging technology of optogenetics, and discussed the ethical and theoretical implications of optogenetics and other technologies. Although technologies such as optogenetics are rapidly increasing our understanding of the brain, we must as humans protect ourselves from potential abuse. With the rapid milieu of research backing optogenetics as an efficacious tool, it may not be long before researchers use it on human volunteers. One may observe the imminent release of Neuralink and other neural implants and imagine a day when optogenetics replaces these technologies to provide widespread technological benefits to society. In moving forward, ethical discussions of optogenetics and CRISPR are paramount to protect humans from experimental abuse and unwanted subjective changes in self-image. 


\section{REFERENCES}

Bartucca, S. (2018). The Most Complicated Object in the Universe. today.uconn.edu/2018/03/ complicated-object-universe

Baumgartner, H. M., Cole, S. L., Olney, J. J., \& Berridge, K. C. (2020). Desire or dread from nucleus accumbens inhibitions: Reversed by same-site optogenetic excitations. The Journal of Neuroscience, 40(13), 2737-2752. https://doi.org/10.1523/jneurosci.2902-19.2020

Bishop, M., Elder, T., \& Heath, R. (1963). Intracranial self-stimulation in man. Science, 140(3565). www.jstor.org/stable/1710935

Boyden, E. S. (2011). A history of optogenetics: The development of tools for controlling brain circuits with light. F1000 Biology Reports, 3(11). https://doi.org/10.3410/b3-11

Britten, K. H. (2002). Area MST and heading perception in macaque monkeys. Cerebral Cortex, 12(7), 692-701. https://doi.org/10.1093/cercor/12.7.692

de Haan, S., Rietveld, E., Stokhof, M., \& Denys, D. (2017). Becoming more oneself? Changes in personality following DBS treatment for psychiatric disorders: Experiences of OCD patients and general considerations. PLOS ONE, 12(4), e0175748. https://doi.org/10.1371/ journal.pone. 0175748

Deisseroth, K., Feng, G., Majewska, A. K., Miesenbock, G., Ting, A., \& Schnitzer, M. J. (2006). NextGeneration Optical Technologies for Illuminating Genetically Targeted Brain Circuits. Journal of Neuroscience, 26(41), 3863-3806. https://doi.org/10.1523/jneurosci.3863-06.2006

Delaloye, S., \& Holtzheimer, P. (2014). Deep brain stimulation in the treatment of depression. Dialogues in Clinical Neuroscience, 16, 1. www.researchgate.net/publication/ 261737714_Deep_brain_stimulation_in_the treatment_of_depression

Faria, M. (2013). Violence, mental illness, and the brain - A brief history of psychosurgery: Part 1 From trephination to lobotomy. Surgical Neurology International, 4(1), 49. https://doi.org/ $\underline{10.4103 / 2152-7806.110146}$

Gilbert, F., Harris, A. R., \& Kapsa, R. M. I. (2014). Controlling brain cells with light: Ethical considerations for optogenetic clinical trials. AJOB Neuroscience, 5(3), 3-11. https://doi.org/ $\underline{10.1080 / 21507740.2014 .911213}$

Gilbert, F., Harris, A. R., \& Kidd, M. (2021). Burnt in your memory or burnt memory? Ethical issues with optogenetics for memory modification. AJOB Neuroscience, 12(1), 22-24. https://doi.org/ $\underline{10.1080 / 21507740.2020 .1866104}$

Kravitz, A. V., Freeze, B. S., Parker, P. R. L., Kay, K., Thwin, M. T., Deisseroth, K., \& Kreitzer, A. C. (2010). Regulation of parkinsonian motor behaviours by optogenetic control of basal ganglia circuitry. Nature, 466(7306), 622-626. https://doi.org/10.1038/nature09159

MacDougall, M., \& Sharma, S. (2020). Physiology, Chemoreceptor Trigger Zone. Physiology, Chemoreceptor Trigger Zone - StatPearls - NCBI Bookshelf. nih.gov

Marzullo, T. (2017). The missing manuscript of Dr. Jose Delgado's radio controlled bulls. The Journal of Undergraduate Neuroscience Education, 15(2). https:/www.ncbi.nlm.nih.gov/pmc/articles/ $\underline{\text { PMC5480854/ }}$

Mathews, D. J. H. (2011). Deep brain stimulation, personal identity and policy. International Review of Psychiatry, 23(5), 486-492. https://doi.org/10.3109/09540261.2011.632624

Memi, F., Ntokou, A., \& Papangeli, I. (2018). CRISPR/Cas9 gene-editing: Research technologies, clinical applications and ethical considerations. Seminars In Perinatology, 42(8), 487-500. https://doi.org/10.1053/j.semperi.2018.09.003 
Neely, D. (2021). Project MKULTRA: How the CIA used the cold war to commit horrors on US citizens. Phi Alpha Theta Pacific Northwest Conference. : pdxscholar.library.pdx.edu/cgi/ viewcontent.cgi? article $=1027 \&$ context $=$ pat_pnw

Pham, U., Solbakk, A.-K., Skogseid, I.-M., Toft, M., Pripp, A. H., Konglund, A. E., Andersson, S., Haraldsen, I. R., Aarsland, D., Dietrichs, E., \& Malt, U. F. (2015). Personality Changes after Deep Brain Stimulation in Parkinson's Disease. Parkinson's Disease, 2015, 1-7. https://doi.org/10.1155/ $\underline{2015 / 490507}$

Servitje, L. (2018). Of drugs and droogs: Cultural dynamics, psychopharmacology, and neuroscience in Anthony Burgess's A Clockwork Orange. Literature and Medicine, 36(1), 101-123. https://doi.org/10.1353/lm.2018.0004

Shao, J., Wang, M., Yu, G., Zhu, S., Yu, Y., Heng, B. C., Wu, J., \& Ye, H. (2018). Synthetic far-red light-mediated CRISPR-dCas9 device for inducing functional neuronal differentiation. Proceedings of the National Academy of Sciences, 115(29), E6722-E6730. https://doi.org/10.1073/ pnas. 1802448115

Shultz, D., \& Schultz, S. (2019). Theories of Personality. Cengage Learning.

Van Essen, D. (2012). The human connectome project: A data acquisition perspective. NeuroImage, 62. https://doi.org/10.1016/j.neuroimage.2012.02.01

Williams, J. C., \& Entcheva, E. (2015). Optogenetic versus electrical stimulation of human cardiomyocytes: Modeling insights. Biophysical Journal, 108(8), 1934-1945. https://doi.org/ 10.1016/j.bpj.2015.03.032

Ye, Z. J., Zhang, X. Y., Liang, J., \& Tang, Y. (2019). The challenges of medical ethics in china: Are gene-edited babies enough? Science and Engineering Ethics, 26(1), 11948-11019. https://doi.org/ 10.1007/s11948-019-00090-7 